\title{
Impact of Health Education on Usage of Malaria Prevention Methods and Reported Malaria in Western Kenya
}

\author{
Jomama One Jomama Lual, ${ }^{1, *}$, Constatin Loum², Richard Mugga ${ }^{2}$ \\ ${ }^{1}$ University Medical Clinic Department, Great Lakes University of Kisumu, Kenya \\ ${ }^{2}$ Tropical Institute of Community Health Faculty, Great Lakes University of Kisumu, Kenya
}

Copyright $\mathrm{O} 2016$ by authors, all rights reserved. Authors agree that this article remains permanently open access under the terms of the Creative Commons Attribution License 4.0 International License

\begin{abstract}
Some of the expected impacts of households' health education on usage of malaria prevention methods include increase in use of insecticide treated bed nets and subsequent reduction in households' reported malaria. This study intended to measure impacts of health education on usage of insecticide treated nets and reported malaria, from November 2012 to June 2013, among rural households in five districts in Western Kenya. At baseline, 1,898 responded to questions on ITN use and 1,016 households responded at end line. At baseline 1,083 households responded to question on households' reported malaria whereas 1,002 responded at end line. Statistical analysis was done using SPSS version 17.0. This study found that there was significant increase in ITNs use (baseline 90.3\% $(1714 / 1898)$; end line 94.9\% (964/1016); $p$-value $=0.0001$; $\mathrm{OR}=0.36 ; 95 \% \mathrm{CI}=0.4-0.7)$. However, it found that there was insignificant reduction in households' reported malaria (baseline 61.7\% (668/1083); end line 58.6\% (587/1002); $\mathrm{p}$-value $=0.149 ; \mathrm{OR}=1.14 ; 95 \%$ CI $0.95-1.36$. The study concluded that, while households' health education resulted in a significant increase in use of insecticide treated nets, significant increase in ITN usage did not significantly lower households' reported malaria in Five Districts in Western Kenya.
\end{abstract}

Keywords Malaria, Households' Health Education, Impact on Reported Malaria, Use of Insecticide Treated Bed Nets

\section{Introduction}

According to WHO (1), globally presumed and confirmed cases of malaria were $87,579,309$. Out of this alarming figure, Africa alone had 79, 381, 896. The confirmation of malaria showed precisely the same thing: out of $26,313,177$ malaria slides and RDT positive patients globally; 22, 319, 014 are from African region.

At Lakeside, malaria transmission is common among the communities (2) though the level of endemicity of malaria in Kenya varies from region to region.

Malaria, being one of the most common preventable diseases in Kenya, contributes to the high disease burden on the population. Its effects are not only felt at individual and household levels, but also within the social and economic spheres of a community. Malaria is transmitted mainly at the community level through what community members do or what they fail to do. As such, a community-based approach to enable households and communities take active roles in health and development issues, was necessary, as set out in Community Strategy (3).

At the community level, malaria prevention activities focus on effective communication that is aimed at behaviour change and basic care (3). The behaviour change and basic care are believed to be attained through the Community Health Strategy that has elaborate governance structures for the coordination of the functioning of different actors, including Community Health Extension Workers, Community Health Committees and Community Health Workers. A Community Health Committee is the first structure in healthcare delivery framework. Using evidence-based plan, health committees organize actions for health grounded in their own capacities (3).

Moreover, a Community Health Worker is a person chosen by his own community through the Community Health Committee and trained on health and development issues to act as a community mobilizer and motivator for positive change. He, therefore, acts as the link between the community and the other levels of health system. Community health workers are allocated specific coverage targets on populations in specified geographic and technical areas, and trained and supervised by a trained Community Health Extension Worker (3). 
As with many vector-borne diseases, control of malaria relies on a combination of case treatment, vector eradication, and personal protection from vector bites, e.g. insecticide (permethrin) treated nets. According to (4), the use of insecticide-treated nets (ITNs) is one of the most feasible weapons available for malaria control in Africa today. One of the important requirements for ITN use is regular re-treatment at an appropriate time. As a response to the low re-treatment rate when the ITN users are expected to purchase insecticide and re-treat their nets, manufacturers have developed long-lasting insecticidal nets (LLIN) that are pre-treated in the factory, and are claimed to require no further treatment during their lifespan (4). Long lasting insecticide treated nets (LLINs) have been advocated as an effective tool for prevention and control of malaria. Olyset net was the first LLINs which became commercially available and obtained WHO approval (5).

The number of ITNs procured in 2012 globally (66 million) is far lower than in 2011 (92 million) and 2010 (145 million). With the average useful life of ITNs estimated to be 2 to 3 years, ITN coverage is expected to decrease if ITNs are not replaced in 2013 (6).

Long-lasting, insecticide-treated nets (LLINs) are one of the most efficacious preventive interventions against malaria morbidity and mortality available and form a cornerstone of the Roll Back Malaria (RBM) Partnership's scaling-up for impact strategy to reduce malaria-related mortality by $75 \%$ from 2000 levels by 2015. To achieve this level of impact, RBM has set the target of reaching and sustaining $80 \%$ universal coverage with LLINs, meaning that $80 \%$ of all members of populations at risk of malaria should be sleeping under an ITN. The RBM strategic plan recommends that the $80 \%$ universal coverage target is achieved using a combination of campaigns and continuous channels such as routine antenatal clinics (ANC) and the expanded programme of immunization (EPI) for LLIN delivery, the so-called "catch-up" and "keep-up" approach (7).

It is in this context that a community health worker provides, as one of his functions, household health education on prevention of malaria. So increase in usage of insecticide treated nets and subsequent reduction in reported malaria among households is one of the expected health outcomes of households' health education.

Therefore, the specific objective of this study was to determine the impact of health education on usage of insecticide treated bed nets and subsequent reported malaria among households in five districts in Western Kenya. The five districts were Butere, Nambale, Nyakach, South Rachuonyo and Suba.

\section{Materials and Methods}

\subsection{Study Design}

This study used a quasi-experimental study design.

\subsection{Study Site}

This study was done in five districts in Western Kenya, namely Butere, Nambale, Nyakach, South Rachuonyo and Suba. The baseline cross sectional survey was done in November 2012 and the end line cross sectional survey in June 2013.

\subsection{Study Population}

This study population was all households of Butere, Nambale, Nyakach, South Rachuonyo and Suba districts.

\subsection{Target Population}

The target population for this study was households with children less than five years or pregnant mothers. The unit of observation was a household represented by household head.

\subsection{Unit of Analysis}

The unit of analysis was a household.

\subsection{Sampling Technique}

During the survey, multi-stage cluster sampling technique was used where all the five (5) districts and twenty nine (29) divisions in the Western Kenya were included. In the first stage three sub-locations were randomly selected from each division, 5 villages were further randomly selected using simple random technique from each sampled sub-location. A total of 20 households were to be covered per village. A starting household was identified at the centre of each village by the supervisor and the enumerator. Random sampling was applied in order to select households to interview. Data was collected using household survey questionnaires by trained enumerators.

\subsection{Sample Size Determination}

Sample size determination was based on the World Health Organization (WHO) EPI 30 by 10 cluster methodology. Three divisions from each district were randomly picked; a total number of 29 sub-locations were in turn selected using the lottery method. Five (5) villages per sub-location were randomly selected where 20 households were to be covered bringing to 100 number of households per sub-location, hence bringing the number to 2900 households'.

\subsection{Sample Size Calculation}

For this study, the villages were the clusters while the households were the cumulative populations, considering also the number of the Sub-locations. Therefore; Sample size (n): 5 "clusters" of 20 households each $(\mathrm{n}=100)$. "Cluster" = $29 \times 100=2900$ 


\subsection{Inclusion Criteria}

Households with children under the age of 5 years old and pregnant mothers preceding the survey were eligible for the study.

\subsection{Exclusion Criteria}

This study excluded households who did not have children under the age of five years old and pregnant mothers prior to the study.

\subsection{Data Collection Tools and Quality Assurance}

Data was collected through structured questionnaires which was written in English and then translated into Luo to ensure harmonization and consistency in terms of message delivery. Pre-test was done in the nearby community for clarity, acceptability, flow and consistency of the study. The questionnaire was used to collect quantitative data from the households.

Data collection was done by trained enumerators who had a minimum of Diploma in Community health and Development or above and at least two years experienced in surveys. They underwent training using the English version and the Luo translation templates for a period of three days. Data collection teams worked in groups of ten enumerators under one supervisor and two research assistants.

\subsection{Data Collection Procedure}

A starting household was identified at the centre of each village by the supervisor and the enumerator. During the survey, each enumerator created rapport with each respondent and explained to them the purpose of the study before administering the questionnaire. Consent seeking was then sort before interviews. A total of 1, 898 households were interviewed at baseline, and 1,016 households at end line. Quantitative data was then collected through structured questions and was collected for period of 2 days. Each enumerator interviewed a total of 20 households. By the end of each day, the supervisor would review the questionnaires for consistency and completeness of data. Finally, all the questionnaires were sorted and serialized.

\subsection{Data Management}

Immediately after field work, data was entered on daily basis by the data clerks. Consistency and completeness checks were done and data cleaned. Information from the questionnaires was electronically entered in Statistical Package for Social Sciences (SPSS) version 17.0 programme. All the hard copies of all questionnaires were used for counter checking the information electronically entered. Each tool was entered individually and no merging was done during the entry.

\subsection{Data Analysis}

The data collected at baseline in November 2012 were compared with those collected at end line in June 2013, a period of eight months. To determine impact of households' health education on usage of insecticide treated nets and reported malaria, Chi Square test was used where p-value of $<0.05$ were considered significant results. Data were compared per district and overall. In this case, odds ratio and $95 \%$ confidence interval were reported to show strength of associations. Statistical analysis was done using SPSS version 17.0 and STATA version 10.

\subsection{Ethical Consideration}

The study was approved by Great Lakes University of Kisumu Ethical Committee and further approval was obtained from Ministry of Public Health and Sanitation. Consent was given before interview was conducted and confidentiality of collected data was ensured.

\subsection{Expected Application of Study Findings}

The findings of this study will be used by all health workers in the five districts to improve on the prevention of malaria by considering multiple interventional approaches to prevention of malaria among households. The findings will moreover, stimulate further research on why significant increase in use of insecticide treated nets does not result in a significant reduction in prevalence of malaria among households in the Five Districts in Western Kenya.

\section{Findings}

\subsection{Impact of Health Education on Usage of Insecticide Treated Nets}

This study found that, overall; there was impact of health education usage of insecticide treated nets in five districts in Western Kenya from $90.3 \%(1714 / 1898)$ at baseline to $94.9 \%$ (964/1016) at end line. An increase of $4.6 \%$ in use of insecticide treated nets among the rural households over a period of eight months. This observed increase in use of insecticide treated nets was statistically significant with $\mathrm{p}$-value $=0.001 ; \mathrm{OR}=0.36 ; 95 \% \mathrm{CI}=0.4-0.7$. This means that routine households' health education may significantly increase usage of insecticide treated nets over a period of eight months. The odds ratio of 0.36 is protective: the more household uses insecticide treated nets, the less likely the reported malaria.

\subsection{Impact of Health Education on Reported Malaria}

Besides, there was impact of households' health education on reported malaria in five districts in Western Kenya from $61.7 \%(668 / 1083)$ at baseline to $58.6 \%(587 / 1002)$ at end 
line. A reduction of $3.1 \%$ in reported malaria among rural households, over a period of eight months. However, this observed reduction in reported malaria was statistically insignificant with $\mathrm{p}$-value $=0.149 ; \mathrm{OR}=1.14 ; 95 \% \mathrm{CI}$ 0.95-1.36. This finding means that routine households' health education may not significantly lower reported malaria over a period of eight months in five districts in western Kenya. The odd ratio of 1.14 means increasing risk of reporting malaria.

\section{Discussions}

In this study there was a significant impact of health education on use of insecticide treated nets in five districts in Western Kenya. The significant impact could be attributed to one important factor namely, household health education that is given every month by community health workers on proper use of insecticide treated nets as a result of introduction of community strategy in all the districts in Western Kenya. This is supported by the nature of impact as seen in the case of Nyakach because community health strategy had just commenced and so only in one location.

Moreover, this finding clearly shows what a great impact could be achieved once the gap between the community and health system is closed by use of community health workers to provide routine households' health education. Furthermore, it reinforces the widely held belief in increasing use of insecticide treated nets as malaria prevention method.

The result of this study agrees with findings of a nationwide cross-sectional survey conducted six months post-campaign in Madagascar by Thawani and colleaques (8) to reduce morbidity and mortality in children under the age of five years, due to malaria. In households with at least one LLIN, the proportion of children under five years of age who slept under an LLIN the previous night was high in both areas with and without integration; $94.6 \%$ in districts with LLIN integration and $90.0 \%$ in districts without integration.

It is, also, consistent with results of a cross sectional study to measure LLIN usage and ownership conducted by Médecins Sans Frontières (9) in Bo and Pujehun. In 94.1\% of the households reporting LLINs, the nets were observed hanging correctly over the beds. Of the 1135 hanging LLINs, $75.2 \%$ had no holes or 10 or fewer finger-size holes. Of the 4997 household members, $67.2 \%$ reported sleeping under an LLIN the night before the study, including $76.8 \%$ of children under 5 years.

Moreover, the finding of this study agrees with results of a study done by Fernando and colleagues (10) in Sri Lanka. A significant increase was seen in the proportion of households using LLINs the previous night during the high transmission season (96\%) compared with the low transmission season $(90 \%)$ for all three ethnic groups. The percentage of children under five who slept under a LLIN increased from $75 \%$ during the low transmission season to $90 \%$ during the high transmission season.

The finding is consistent with results of a cross sectional households and health facility surveys conducted in Tanzania by (Bonner et al., 2011). The findings of a study conducted to determine the LLIN use rate, and to identify key factors associated with LLIN use in households by Ouedraogo et al., (11) also seem to agree: when an association was demonstrated, the relative risk (RR) was calculated with the $95 \%$ confidence interval. The LLIN use rate was $81.7 \%$ in children under 5 years Ouédraogo et al., (11).

However, in this study there was insignificant impact on reported malaria. This means that a significant impact of households' health education on use of insecticide treated nets may not result in a significant impact on reported malaria. This is clearly the opposite of what was expected. If the impact on usage of insecticide treated nets is significant among same target population, then the expected impact on reported malaria should be equally significant.

This result is inconsistent with the finding of a double blind randomized placebo controlled clinical trial conducted in Bolivian Amazon to determine the effectiveness in reducing malaria (12) in which was found a highly significant $80 \%$ reduction in episodes of $\mathrm{P}$ vivax in the group that used treated nets and repellent (incidence rate ratio 0.20 , $95 \%$ confidence interval 0.11 to $0.38, \mathrm{P}<0.001$ ). Numbers of P. falciparum cases during the study were small and, after adjustment for age, an $82 \%$ protective effect was observed, although this was not significant $(0.18,0.02$ to $1.40, \mathrm{P}=0.10)$. Reported episodes of fever with any cause were reduced by $58 \%$ in the group that used repellent $(0.42,0.31$ to 0.56 , $\mathrm{P}<\mathrm{O} .001)$.

He concluded that Insect repellents can provide protection against malaria. In areas where vectors feed in the early evening, effectiveness of treated nets can be significantly increased by using repellent between dusk and bedtime.

Similarly, in the Olyset net study area, malaria incidence was reduced by $96.6 \%$ and $64.8 \%$ in the village with Olyset nets and in the villages with untreated nets, respectively. During intervention period, there was a reduction of $93.2 \%$ in malaria incidence in Olyset net area as compared to the untreated area (13).

Muller and colleagues (14) conducted a randomized control trial in Burkina Faso, on effects of ITNs during infancy in an African area of intense malaria transmission. The results found that an overall significantly lower plasmodium falciparum malaria morbidity in group using ITNs than the group not using ITNs.

A study by Hawley and colleaques (15) on effects of ITNs on nearby households both with and without ITNs was performed in Asembo, Kenya. The findings illustrated a protective effect of ITNs in compound lacking ITNs located within 3000 metres of a compound with ITNs for child mortality, moderate anaemia, high density parasitemia, and haemoglobin levels.

The limitation in the present study was that malaria testing 
was not conducted by the researchers during data collection: but, the diagnosis of malaria was confirmed using available patient records. Further research would be required to investigate the reasons for insignificant impact on reported malaria in five Districts in Western Kenya.

\section{Conclusions}

Household health education resulted in a significant impact on usage of insecticide treated nets in five Districts in Western Kenya. However, the significant impact of households' health education did not result in the subsequent significant impact on reported malaria in five Districts in Western Kenya.

\section{Acknowledgements}

We are grateful to the late Prof. David Wasawa; Sarah Awak Jok; Ayak Jomama and Kulang Jomama for their inspiration and encouragement.

\section{REFERENCES}

[1] World Health Organization (2012). World Malaria Report. World Health Organization, Geneva

[2] Kenya Ministry of Health (2006). Malaria Treatment Guidelines. Ministry of Health. Nairobi.

[3] Division of Community Health Services, (2011) Integrated Curriculum for Training Community Health Workers in Kenya, MOPHS Nairobi.

[4] Msangi, S., Lyatuu, E., Masenga C., Kihumo, E. (2008). The effects of washing and duration of long lasting insecticidal nets (PermaNets) on insecticidal effectiveness. Acta Tropica, 107 (1):43-47.

[5] .Ahmadi M.S. Vatandoost H, Shaeghi M, Raeisi A, Abedi F, Eshraghian M. R, Madani A, Safari R, Oshaghi M. A, Abtahi M, Hajjaran H. (2012): Field evaluation of permethrin long lasting insecticide treated nets for malaria control in an endemic area, south east of Iran Acta Tropica 123 (3): 146-153

[6] World Health Organization (2012). World Malaria Report. World Health Organization, Geneva.

[7] Okello L. C., Paintain L. S., Webster J., Hanson K. and Lines J. (2012) From intervention to impact: modelling the potential mortality impact achievable by different long lasting, insecticide treated nets strategies. Malaria Journal 11 (327):

\section{$1475-2875$}

[8] Thawani N. Manisha A. Kulkarni M. A. and Sohan S. (2009). Factors Associated with Coverage and Usage of Long-Lasting Insecticidal Nets in Madagascar, Journal of Tropical Medicine 2009 (2009). http://dx.doi.org/10.1155/20 $09 / 451719$

[9] Medicins San Frontieres (2010) Long lasting insecticide treated nets usage in Eastern Serrileon. Tropical medicine and international health, 15 (4): 480-8.

[10] Fernando S. D. Abeyasinghe R.R., Galappaththy G. N. L., Gunawardena N. and Rajapaksa L. C (2008): Community factors affecting long-lasting impregnated mosquito net use for malaria control in Sri Lanka Transactions of the Royal Society of Tropical Medicine and Hygiene 102HYPERLINK "http://www.sciencedirect.com/science/journal/00359203/10 2/11" (HYPERLINK "http://www.sciencedirect.com/science /journal/00359203/102/11"11):1081-1088

[11] Ouédraogo T. L., Ouédraogo I, Yaméogo A. Ouédraogo V. (2013): Determinants of long-lasting insecticidal net use in Burkina Faso after a mass distribution in the Diébougou health district Revue HYPERLINK "http://www.sciencedire ct.com/science/journal/03987620"d'Épidémiologie HYPERL INK "http://www.sciencedirect.com/science/journal/039876 20" HYPERLINK "http://www.sciencedirect.com/science/jo urnal/03987620"et HYPERLINK "http://www.sciencedirect. com/science/journal/03987620" de Santé HYPERLINK "http://www.sciencedirect.com/science/journal/03987620"Pu blique 61 HYPERLINK "http://www.sciencedirect.com/scie nce/journal/03987620/61/2" HYPERLINK "http:/www.scie ncedirect.com/science/journal/03987620/61/2"(2): 121-127

[12] Hill, N., Lenglet A., Arnez A. M., Carneiro I. \& Roberts. (2007). Plant based insect Repellent and Insecticide Treated Bed Nets to protect against Malaria in areas of early Evening Biting Vectors: Double Blind Randomised placebo Controlled Clinical trial in the Bolivian Amazon. British Medical Journal, 335 (7628): 1023-1025

[13] Ahmadi M.S. Vatandoost H, Shaeghi M, Raeisi A, Abedi F, Eshraghian M. R, Madani A, Safari R, Oshaghi M. A, Abtahi M, Hajjaran H. (2012): Field evaluation of permethrin long lasting insecticide treated nets for malaria control in an endemic area, south east of iran Acta Tropica 123 (3): $146-153$

[14] Muller, O., Traoré, C., Kouyaté, B., Yé, Y., Frey, C., Coulibaly, B., Becher, H., (2006) Effects of insecticide-treated bed nets during early infancy in an African area of intense malaria transmission: a randomized controlled trial. Bulletined of World Health Organization, 84 (2).

[15] Hawley, W. A, Phillips-Howard P. A, ter Kuile F. O, Terlouw D. J, Vulule J. M, Ombok M, Nahlen B. L, Gimnig J. E, Kariuki S. K, Kolczak M. S, Hightower A. W. (2003). Community-wide effects of permethrin-treated bed nets on child mortality and malaria morbidity in western Kenya. American Journal of Tropical Medicine and Hygiene, 66 (4):121-7. 